\title{
Efeitos de superfície no espectro do Modelo de Timoshenko
}

\author{
Julio R. Claeyssen, $\quad$ Leticia Tonetto, \\ Instituto de Matemática, UFRGS, \\ 91509-900, Porto Alegre, RS \\ Programa de Pós-Graduação em Matemática Aplicada - PPGMap, UFRGS, \\ 91509-900, Porto Alegre, RS \\ Elba Bravo-Asenjo \\ Centro de Ciência e Tecnologia, UENF \\ 28013-602, Campos, RJ \\ E-mail: julio@mat.ufrgs.brｌtonetto@gmail.com elba.bravo@gmail.com
}

\begin{abstract}
Resumo: É realizado um estudo das vibrações transversais livres de vigas em nanoescala, através da inclusão de efeitos de superfície às equações do modelo clássico de Timoshenko. O problema de autovalor correspondente as amplitudes espaciais é descrito por uma equação diferencial matricial singular de terceira ordem, sujeita a condições de contorno. As frequências naturais e modos são caracterizadas, no caso não defeituoso para vigas bi-apoiadas e fixa-livres. É observado que os efeitos de superfície influenciam as frequências naturais de vigas apenas em nanoescala, quando o comprimento cresce de nanometros para micrometros, os efeitos de superfície desaparecem e os resultados convergem para as frequências naturais do modelo clássico de Timoshenko.
\end{abstract}

Palavras-chave: Vibração de nanovigas, Modelo de Timoshenko, Efeitos de superfície.

\section{Introdução}

Atualmente tem crescido o interesse no desenvolvimento de sistemas nano-eletro-mecâmicos (NEMS). Esses podem ser sensores, atuadores, máquinas, e eletrônicos caracterizados em nanoescala, cujas configurações podem fazer uso de micro e nanovigas. O interesse das ciências em geral por essas escalas menores, têm também impulsionado o desenvolvimento de tecnologias tais como microscópios de alta precisão, por exemplo microscópios de força atômica, e transdutores micro-eletro-mecânicos como plataforma para sensores químicos e biológicos [9], [6], [3], [7].

Quando os comprimentos das escalas associadas são suficientemente pequenos, a aplicabilidade dos modelos contínuos clássicos pode não ser apropriada. Esse tópico tem sido discutido na literatura com a proposta de modelos que incluem diferentes efeitos, tais como efeitos de superfície e efeitos não locais, que modificam as teorias convencionais de vibração de vigas em micro e nanoescala [1], [8], [10].

Efeitos de superfície podem desempenhar um papel importante nas propriedades físicas de materiais e estruturas, visto que os átomos dentro de uma fina camada próxima da superfície podem interagir de uma maneira local diferente do restante dos átomos na parte principal da estrutura (bulk). Sendo assim as propriedades físicas e respostas mecânicas das superfícies irão ser distintas daquelas do restante da viga [1], [10].

Nesse trabalho será apresentado um estudo do problema de autovalor associado a um modelo de Timoshenko incluindo efeitos de superfície [6]. Devido a esses efeitos o problema de autovalor é descrito por uma equação diferencial matricial singular de terceira ordem sujeita a condições 
de contorno. Ainda que o polinômio característico dessa equação matricial tenha a mesma forma do correspondente ao modelo clássico de Timoshenko, a dependência dos coeficientes com respeito ao autovalor é mais complexa devido aos efeitos mencionados. A multiplicidade dos autovalores será caracterizada em termos de parâmetros de superfície críticos. Utilizando o método espectral de Euler serão determinadas soluções da equação diferencial matricial para o caso não-defeituoso e o caso defeituoso correspondente a amplitudes espaciais constantes. Modos e frequências naturais são então obtidas para vigas do tipo bi-apoiadas e fixa-livre com efeitos de superfície. Serão apresentadas comparativamente as frequências naturais correspondentes aos modelos com e sem tais efeitos, em nano e micro escala.

\section{Modelo de Timoshenko incluindo efeitos de superfície}

Consideramos uma viga de material isotrópico, com seção transversal retangular, de comprimento $L$, largura $b$, altura $H=2 h$. O momento de inércia da área da seção tranversal é $I=\frac{2 b h^{3}}{3}$, sendo $A=2 b h$ a área da seção transversal. Diferentemente do modelo clássico, no modelo proposto em [6], o qual é baseado no modelo contínuo de Gurtin-Murdoch [5], considerase que a viga tem uma superfície elástica de espessura nula perfeitamente colada ao seu volume principal (bulk). As equações que governam a deflexão flexural e o ângulo de rotação da viga, $w(t, x)$ e $\phi(t, x)$, respectivamente, são dadas por

$$
\begin{aligned}
& \left(\rho A+\rho_{0} s^{*}\right) w_{t t}-\left(\kappa G A+\tau_{0} s^{*}\right) w_{x x}+\kappa G A \phi_{x}=0 \\
& \left(\rho I+\rho_{0} I^{*}\right) \phi_{t t}-\left(E I+\left(2 \mu_{0}+\lambda_{0}\right) I^{*}\right) \phi_{x x}-\frac{2 \nu I \rho_{0}}{H} w_{x t t}+\frac{2 \nu I \tau_{0}}{H} w_{x x x}-\kappa G A\left(w_{x}-\phi\right)=0
\end{aligned}
$$

De forma simplificada consideramos

$$
\begin{aligned}
& c_{m} w_{t t}-a_{m} w_{x x}+a \phi_{x}=0, \\
& e_{m} \phi_{t t}-b_{m} \phi_{x x}-I^{0} w_{x t t}+I^{1} w_{x x x}-a\left(w_{x}-\phi\right)=0,
\end{aligned}
$$

$\operatorname{com} c_{m}=\rho A+\rho_{0} s^{*}, a_{m}=\kappa G A+\tau_{0} s^{*}, a=\kappa G A, e_{m}=\rho I+\rho_{0} I^{*}, \quad I^{0}=\frac{2 \nu I \rho_{0}}{H}, \quad I^{1}=\frac{2 \nu I \tau_{0}}{H}$.

Os parâmetros materiais da parte principal da viga são $E, \nu$, e $\rho$, que representam o Módulo de Young, o coeficiente de Poisson e a densidade de massa, respectivamente.Temos também os parâmetros referentes a superfície da viga $\lambda_{0}, \mu_{0}, \tau_{0}, \rho_{0}$, sendo que os dois primeiros representam as constantes de Lamé, e os dois últimos são a tensão residual de superfície e a densidade de massa da superfície. $I^{*}$ é o momento de inércia correspondente a superfície, e análogo ao momento de inércia $I$ da parte principal da viga, utilizaremos $I^{*}=2 b h^{2}+4 h^{3} / 3$, e $s^{*}=2 b . \kappa$ é o coeficiente de correção de cisalhamento.

Observamos que tal modelo se reduz ao modelo clássico de Timoshenko quando os efeitos de superfície são desconsideramos.

\section{Formulação matricial}

As equações descritas em (2) pode ser escritas na seguinte forma matricial compacta

$$
\mathbf{M} \ddot{v}+\mathbf{K} v=0
$$

sendo

$$
\mathbf{M}=M_{0}+M_{1}, \quad M_{0}=\left(\begin{array}{cc}
c_{m} & 0 \\
0 & e_{m}
\end{array}\right), \quad M_{1}=K_{4} \frac{\partial}{\partial x}, \quad K_{4}=\left(\begin{array}{cc}
0 & 0 \\
-I^{0} & 0
\end{array}\right)
$$


$\mathbf{K}=K_{1} \frac{\partial^{3}}{\partial x^{3}}+K_{2} \frac{\partial^{2}}{\partial x^{2}}+K_{3} \frac{\partial}{\partial x}+K_{5}$

$$
K_{1}=\left(\begin{array}{cc}
0 & 0 \\
I^{1} & 0
\end{array}\right), \quad K_{2}=\left(\begin{array}{cc}
-a_{m} & 0 \\
0 & -b_{m}
\end{array}\right), K_{3}=\left(\begin{array}{cc}
0 & a \\
-a & 0
\end{array}\right), \quad K_{5}=\left(\begin{array}{cc}
0 & 0 \\
0 & a
\end{array}\right) .
$$

E então, podemos reescrever (3) como

$$
\left(\begin{array}{cc}
c_{m} \frac{\partial^{2}}{\partial t^{2}}-a_{m} \frac{\partial^{2}}{\partial x^{2}} & a \frac{\partial}{\partial x} \\
-I^{0} \frac{\partial^{3}}{\partial t^{2} \partial x}+I^{1} \frac{\partial^{3}}{\partial x^{3}}-a \frac{\partial}{\partial x} & e_{m} \frac{\partial^{2}}{\partial t^{2}}-b_{m} \frac{\partial^{2}}{\partial x^{2}}+a
\end{array}\right) \mathrm{v}=0
$$

\section{Problema de Autovalor}

Substituindo as seguintes soluções exponenciais $\mathbf{v}(t, x)=e^{\gamma t} \mathbf{w}(x)$, com $\mathbf{w}(x)=\left(\begin{array}{c}W(x) \\ \Psi(x)\end{array}\right)$, em (3), resulta que a amplitude espacial $\mathbf{w}(x)$ satisfaz a equação diferencial matricial de terceira ordem

$$
\mathbb{R} \mathbf{w}^{\prime \prime \prime}(x)+\mathbb{M} \mathbf{w}^{\prime \prime}(x)+\mathbb{C}(\gamma) \mathbf{w}^{\prime}(x)+\mathbb{K}(\gamma) \mathbf{w}(x)=\mathbf{0},
$$

com

$$
\mathbb{R}=\left(\begin{array}{cc}
0 & 0 \\
I^{1} & 0
\end{array}\right), \mathbb{M}=\left(\begin{array}{cc}
-a_{m} & 0 \\
0 & -b_{m}
\end{array}\right), \mathbb{C}(\gamma)=\left(\begin{array}{cc}
0 & a \\
-\left(\gamma^{2} I^{0}+a\right) & 0
\end{array}\right), \mathbb{K}(\gamma)=\left(\begin{array}{cc}
\gamma^{2} c_{m} & 0 \\
0 & \gamma^{2} e_{m}+a
\end{array}\right)
$$

Vamos considerar soluções de $(7)$ da forma $\mathbf{w}(x)=e^{\beta x} \mathbf{v}, \mathbf{v} \neq 0$. Tais soluções quando substituídas em (7) resultam no problema de autovalor singular

$$
\Gamma(\beta, \gamma) \mathbf{v}=\left(\beta^{3} \mathbb{R}+\beta^{2} \mathbb{M}+\beta \mathbb{C}(\gamma)+\mathbb{K}(\gamma)\right) \mathbf{v}=0
$$

com

$$
\Gamma(\beta, \gamma)=\left(\begin{array}{cc}
-\beta^{2} a_{m} c_{m} \gamma^{2} & \beta a \\
\beta^{3} I^{1}-\beta\left(I^{0} \gamma^{2}+a\right) & -\beta^{2} b_{m}+e_{m} \gamma^{2}+a
\end{array}\right)
$$

Soluções v não-nulas, podem ser obtidas através do cálculo das raízes $\beta$ do polinômio característico $P(\beta, \gamma)=\operatorname{det}\left(\beta^{3} \mathbb{R}+\beta^{2} \mathbb{M}+\beta \mathbb{C}(\gamma)+\mathbb{K}(\gamma)\right)$, satisfazendo a equação

$$
\Delta(\beta, \gamma)=\beta^{4}+g^{2}(\gamma) \beta^{2}-r^{4}(\gamma)=0,
$$

com

$$
g^{2}(\gamma)=\frac{\left(I^{0} a-b_{m} c_{m}-a_{m} e_{m}\right) \gamma^{2}+a^{2}-a a_{m}}{\left(a_{m} b_{m}-a I^{1}\right)}, \quad r^{4}(\gamma)=-\frac{\gamma^{2} c_{m}\left(a+e_{m} \gamma^{2}\right)}{\left(a_{m} b_{m}-a I^{1}\right)} .
$$

A equação (10) tem as seguintes soluções

$$
\beta_{1,2}=\sqrt{\frac{-g^{2}(\gamma)+\sqrt{\left(g^{2}(\gamma)\right)^{2}+4 r^{4}(\gamma)}}{2}}, \quad \beta_{3,4}=\sqrt{\frac{-g^{2}(\gamma)-\sqrt{\left(g^{2}(\gamma)\right)^{2}+4 r^{4}(\gamma)}}{2}} .
$$

Deve ser observado que a equação característica (10)tem a mesma forma da equação característica do modelo clássico de Timoshenko, ainda que os coeficientes sejam diferentes devido aos efeitos de superfície. 


\subsection{Autovalores simples e repetidos}

Se $\beta$ é raiz do polinômio característico $P(\beta, \gamma)=\beta^{4}+g^{2}(\gamma) \beta^{2}-r^{4}(\gamma)$ verificamos que

(1) $\beta$ é uma raiz simples somente se $\beta \neq 0$ e $\beta^{2} \neq \frac{-g^{2}(\gamma)}{2}$. As únicas raízes repetidas são $\beta=0$ e $\beta^{2}=\frac{-g^{2}(\gamma)}{2}$.

(2) Se $\beta=0$ é raiz, ela será dupla se $g^{2}(\gamma) \neq 0$, ou quadrúpla se $g^{2}(\gamma)=0$.

(3) Se $\beta=\sqrt{\frac{-g^{2}(\gamma)}{2}}$ e $\beta=-\sqrt{\frac{-g^{2}(\gamma)}{2}}$ são raízes, com $g^{2}(\gamma) \neq 0$, então serão raízes duplas.

(4) Não existirão raízes triplas.

Tais propriedades decorrem do fato de que $\beta$ é raiz simples se $P(\beta, \gamma)=0$ e $\frac{\partial}{\partial \beta} P(\beta, \gamma) \neq 0$. E $\beta$ terá multiplicidade $2 \leq r \leq 4$ se $P^{(r-1)}(\beta)=0$ e $P^{(r)}(\beta) \neq 0$.

Para que as possíveis raízes repetidas $\beta=0$ e $\beta= \pm \sqrt{\frac{-g^{2}(\gamma)}{2}}$ ocorram é necessário satisfazer certas condições:

- $\beta=0$ é raiz de $P(\beta, \gamma)$, se e somente se $r^{4}(\gamma)=0$. Além disso, $r^{4}(\gamma)=0$ para $\gamma=0$ ou $\gamma=\gamma_{c}$, sendo $\gamma_{c}=\sqrt{\frac{-a}{e m}}$.

- $\beta= \pm \sqrt{\frac{-g^{2}(\gamma)}{2}}, \operatorname{com} g^{2}(\gamma) \neq 0$, é raiz de $P(\beta, \gamma)$ se e somente se $\left(g^{2}(\gamma)\right)^{2}+4 r^{4}=0$, com $r^{4} \neq 0$. Temos que $g^{2}(\gamma)=0$ para $\gamma=\gamma_{g}$, sendo $\gamma_{g}=\sqrt{\frac{-\left(a^{2}-a a_{m}\right)}{\left(b_{m} c_{m}+a_{m} e_{m}-I^{0} a\right)}}$. E vamos denotar $\gamma_{l}$ solução de $\left.g^{2}(\gamma)\right)^{2}+4 r^{4}=0$

Já que as raízes $\beta$ do polinômio característico $P(\beta, \gamma)$ são os autovalores do problema descrito em (8), teremos a seguinte classificação

(I) Teremos 4 autovalores distintos $\beta_{1} \neq \beta_{2} \neq \beta_{3} \neq \beta_{4}$, se e somente se $\gamma \neq 0, \gamma \neq \gamma_{c}$ e $\gamma \neq \gamma_{l}$.

(II) Teremos um autovalor duplo $\beta_{1}=\beta_{2}=0 \neq \beta_{3} \neq \beta_{4}$ quando $\gamma=0$ e $\gamma \neq \gamma_{g}$ ou $\gamma=\gamma_{c}$ e $\gamma \neq \gamma_{g}$.

(III) Temos dois autovalores duplos distintos $\beta_{1}=\beta_{2}=\sqrt{\frac{-g^{2}(\gamma)}{2}} \neq \beta_{3}=\beta_{4}=-\sqrt{\frac{-g^{2}(\gamma)}{2}}$, quando $\gamma=\gamma_{l} \neq \gamma_{c} \neq \gamma_{g} \neq 0$.

(IV) Teremos um autovalor quadrúplo $\beta_{1}=\beta_{2}=\beta_{3}=\beta_{4}=0$ apenas quando $\gamma=\gamma_{g}=0$ ou $\gamma=\gamma_{c}=\gamma_{g}$.

\subsection{Modos de vibração}

No caso de raízes simples $\beta_{1} \neq \beta_{2} \neq \beta_{3} \neq \beta_{4}$, temos que a solução $\mathbf{w}(x)$ de $(7)$ será

$$
\mathbf{w}(x)=c_{1} e^{\beta_{1} x} \mathbf{v}_{1}+c_{2} e^{\beta_{2} x} \mathbf{v}_{2}+c_{3} e^{\beta_{3} x} \mathbf{v}_{3}+c_{4} e^{\beta_{4} x} \mathbf{v}_{4}
$$

onde os autovetores

$$
\mathbf{v}_{j}=\left(\begin{array}{c}
v_{1 j} \\
\left(\frac{\beta_{j}^{2} a_{m}-\gamma^{2} c_{m}}{\beta_{j} a}\right) v_{1 j}
\end{array}\right)
$$

foram obtidos de (8).

No caso em que $\beta=0$ é autovalor duplo temos que $\beta_{1}=\beta_{2}=0$ e $\beta_{3} \neq \beta_{4} \neq 0$, e a solução será 


$$
\mathbf{w}(x)=c_{1} \mathbf{v}_{1}+c_{2}\left(\mathrm{v}_{1} x+\mathrm{b}_{1}\right)+c_{3} \mathrm{v}_{3} e^{\beta_{3} x}+c_{4} \mathbf{v}_{4} e^{\beta_{4} x},
$$

onde $v_{1}$ é autovetor correspondente e $b_{1}$ é um autovetor generalizado cuja forma depende do valor $\gamma=0$ ou $\gamma=\gamma_{c}$.

Analogamente, no caso em que $\beta=0$ é autovalor quadrúpulo tem-se a solução

$$
\mathbf{w}(x)=c_{1} \mathrm{v}_{1}+c_{2}\left(\mathrm{v}_{1} x+\mathrm{b}_{1}\right)+c_{3}\left(\mathrm{v}_{1} \frac{x^{2}}{2 !}+\mathrm{b}_{1} x+\mathrm{b}_{2}\right)+c_{4}\left(\mathrm{v}_{1} \frac{x^{3}}{3 !}+\mathrm{b}_{1} \frac{x^{2}}{2 !}+\mathrm{b}_{2} x+\mathrm{b}_{3}\right),
$$

onde $b_{2}$ e $b_{3}$ são autovetores generalizados para completar a base [4].

O autovalor nulo ocorre para $\gamma=\gamma_{g}=0$ ou $\gamma=\gamma_{c}=\gamma_{g}$. Observa-se que $\gamma_{g}=0$ ocorre quando $\tau_{0}=0$, ou seja, quando a tensão residual de superfície é nula. O caso (III) é similar ao caso de $\beta=0$ duplo e não será analisado.

No caso de autovalores distintos a solução pode ser escrita numa forma trigonométrica e hiperbólica conveniente. Para isso utilizamos $\beta_{1,2}= \pm \varepsilon$ e $\beta_{3,4}= \pm \delta i \mathrm{com}$

$$
\varepsilon=\sqrt{\frac{-g^{2}(\gamma)+\sqrt{\left(g^{2}(\gamma)\right)^{2}+4 r^{4}(\gamma)}}{2}} \text { e } \delta=\sqrt{\frac{g^{2}(\gamma)+\sqrt{\left(g^{2}(\gamma)\right)^{2}+4 r^{4}(\gamma)}}{2}}
$$

E decorre

$$
\mathbf{w}(x)=\left[\begin{array}{cccc}
C_{1} & C_{2} & C_{3} & C_{4} \\
C_{2} K_{a} & C_{1} K_{a} & C_{4} K_{b} & -C_{3} K_{b}
\end{array}\right]\left[\begin{array}{c}
\cosh (\varepsilon x) \\
\sinh (\varepsilon x) \\
\cos (\delta x) \\
\sin (\delta x)
\end{array}\right]
$$

onde

$$
K_{a}=\frac{a_{m} \epsilon^{2}-\gamma^{2} c_{m}}{a \epsilon}, \quad K_{b}=\frac{a_{m} \delta^{2}+\gamma^{2} c_{m}}{a \delta} .
$$

A fim de determinar as constantes na solução $\mathbf{w}(x)$, no caso de uma viga bi-apoiada, devemos considerar as condições de contorno referentes ao deslocamento e momento fletor nulos nas extremidades da viga

$$
\begin{array}{ll}
w(t, 0)=0, & w(t, L)=0, \\
-b_{m} \phi_{x}(t, 0)+I^{1} w_{x x}(t, 0)-I^{0} w_{t t}(t, 0)=0, & -b_{m} \phi_{x}(t, L)+I^{1} w_{x x}(t, L)-I^{0} w_{t t}(t, L)=0 .
\end{array}
$$

Com as condições de contorno em $x=0$, segue que $C_{1}=C_{3}=0$. Com as condições de contorno em $x=L$ obtém-se um sistema da forma $\mathbf{U c}=0$ Para ter solução c não nula, decorre a equação característica

$$
\begin{gathered}
\Delta(\gamma)=\operatorname{det}(\mathbf{U})=\mathbf{0}, \\
\frac{\left(I^{1} a-b_{m} a_{m}\right)\left(\epsilon^{2}+\delta^{2}\right)}{a} \sinh (\epsilon L) \sin (\delta L)=0 \text { ou } \sinh (\epsilon L) \sin (\delta L)=0 .
\end{gathered}
$$

A fim de determinar as frequências naturais considera-se $\gamma=i \omega$ e a equação característica é resolvida, com $\epsilon$ e $\delta$ em termos de $\omega$.

De forma análoga obtemos a equação característica $\Delta(\omega)=\operatorname{det}(\mathbf{U})$ para viga fixa-livre, considerando as condições de contorno 


$$
\begin{aligned}
& w(0)=0, \quad a_{m} w^{\prime}(L)-a \psi(L)=0, \\
& \psi(0)=0, \quad-b_{m} \psi^{\prime}(L)+I^{1} w^{\prime \prime}(L)+I_{0} \omega^{2} w(L)=0 .
\end{aligned}
$$

Na Tabela 1 apresentamos um comparativo entre as frequências naturais para vigas de Timoshenko, com efeitos de superfície e caso clássico, do tipo bi-apoiada e fixa-livre, em nano e micro-escala. São utilizados os parâmetros geométricos [6] para viga em nanoescala

$$
L=50 \times 10^{-9} \mathrm{~m}, \quad H=2 h=6 \times 10^{-9} \mathrm{~m}, \quad b=3 \times 10^{-9} \mathrm{~m} .
$$

E para viga em microescala

$$
L=50 \times 10^{-6} m, \quad H=2 h=6 \times 10^{-6} m, \quad b=3 \times 10^{-6} m .
$$

A viga considerada é feita de Silício, cujos paramêtros materiais são [6]

$$
\begin{gathered}
E=107 G P a, \nu=0.33 \quad \rho=2.33 \times 10^{3} \frac{\mathrm{kg}}{\mathrm{m}^{3}}, \\
\rho_{0}=3.17 \times 10^{-7} \frac{\mathrm{kg}}{\mathrm{m}^{2}}, \mu_{0}=-2.7779 \frac{\mathrm{N}}{\mathrm{m}}, \quad \lambda_{0}=-4.4939 \frac{\mathrm{N}}{\mathrm{m}}, \quad \tau_{0}=0.6056 \frac{\mathrm{N}}{\mathrm{m}} .
\end{gathered}
$$

\begin{tabular}{|c|c|c|c|c|c|c|c|c|}
\cline { 2 - 9 } \multicolumn{1}{c|}{} & \multicolumn{4}{c|}{ Ap.Ap } & \multicolumn{3}{c|}{ F-L } \\
\cline { 2 - 9 } \multicolumn{1}{c|}{ Nano } & \multicolumn{2}{c|}{ Micro } & \multicolumn{2}{c|}{ Nano } & \multicolumn{2}{c|}{ Micro } \\
\hline & com efeitos & classic. & com efeitos & classic. & com efeitos & classic. & com efeitos & classic. \\
\hline Freq & $(\mathrm{GHz})$ & $(\mathrm{GHz})$ & $(\mathrm{MHz})$ & $(\mathrm{MHz})$ & $(\mathrm{GHz})$ & $(\mathrm{GHz})$ & $(\mathrm{GHz})$ & $(\mathrm{GHz})$ \\
\hline $1^{a}$ & 7.0965 & 7.2044 & 7.2042 & 7.2044 & 3.107 & 2.5976 & 19.163 & 19.163 \\
\hline $2^{a}$ & 25.128 & 27.139 & 27.1378 & 27.139 & 14.684 & 15.290 & 33.877 & 33.883 \\
\hline $3^{a}$ & 51.946 & 56.522 & 56.522 & 56.522 & 36.536 & 39.38 & 57.491 & 57.493 \\
\hline $4^{a}$ & 85.331 & 92.764 & 92.757 & 92.764 & 64.660 & 69.95 & 95.817 & 95.818 \\
\hline
\end{tabular}

Tabela 1: Comparativo entre frequências naturais dos modelos com efeitos de superfície e modelo clássico de Timoshenko, em nano e microescala.

\section{Conclusões}

Este trabalho apresenta o estudo de um problema de autovalor singular que resulta de um modelo que modifica o modelo clássico de Timoshenko para vibração de nanovigas, considerando efeitos de superfície. A procura de ondas de natureza plana leva ao estudo de uma equação diferencial matricial singular de terceira ordem sujeita a condições de contorno. Foi caracterizada a multiplicidade dos autovalores em termos de parâmetros de superfície críticos. Foram determinados os autovetores no caso não-defeituoso e caracterizados os autovetores generalizados no caso defeituoso $\beta=0$, com multiplicidade dupla e quádrupla.

$\mathrm{Na}$ Tabela 1 foram apresentadas comparativamente as frequências naturais referentes aos modelos com efeitos de superfície e modelo clássico de Timoshenko, em nano e microescala. Constatamos que quando a viga considerada tem suas dimensões geométricas numa escala micrométrica as frequências naturais calculadas para o modelo incluindo efeitos de superfície tendem aquelas obtidas no caso clássico. Isso evidencia que os efeitos de superfície são significativos apenas na nanoescola. Tal fato foi verificado na literatura para um modelo de Timoshenko também modificado por efeitos de superfície, distinto do modelo apresentado no presente trabalho para condição de contorno do tipo apoiada-apoiada [1], e para condição de contorno do tipo fixa-livre [2]. 


\section{Referências}

[1] S. Abbasion, A. Rafsanjani, R. Avazmohammadi, and A. Farshidianfar. Free vibration of microscaled Timoshenko beam. Applied Physics Letters, 95(14):143122, 2009.

[2] J.R. Claeyssen, L. Tonetto, and T. Tsukazan. Micro-beams with surface and piezoelectric effects in AFM. Conferência Brasileira de Dinâmica, Controle e Aplicações, 2013.

[3] J.R. Claeyssen, T. Tsukazan, L. Tonetto, and D. Tolfo. Modeling the tip-sample interaction in atomic force microscopy with Timoshenko beam theory. Nanoscale Systems: Mathematical Modeling, Theory and Applications, (2)(DOI: 10.2478/nsmmt-2013-0008):124-144, 2013.

[4] R.J. Duffin. Chrystal's theorem on differential equation systems. Journal of Mathematical Analysis and Applications, 8:325-331, 1963.

[5] M.E. Gurtin and A.I. Murdoch. Surface stress in solids. International Journal of Solids and Structures, The University of British Columbia(14(6)):431-440, 1978.

[6] C. Liu. Surface elasticity models for static and dynamic response of nanoscale beams. Master Thesis, The University of British Columbia, 2010.

[7] J.B. Zhou M. Zhang, G. Meng and J.Y. Chen. Nonlinear dynamics and chaos of microcantilever-based tm-afms with squeeze film damping effects. Journal of Mathematical Analysis and Applications, 84(1):64-76, 1998.

[8] B.Bar On, E. Althus, and E.B. Tadmor. Surface effects in non-uniform nanobeams: Continuum vs atomistic modeling. International journal of solids and structures, 47:1243-1252, 2010 .

[9] J.E. Sader. Frequency response of cantilever beams immersed in viscous fluids with applications to the atomic force microscope. Journal of Mathematical Analysis and Applications, 84(1):64-76, 1998.

[10] G.F Wang and X.Q Feng. Effects of surface elasticity and residual surface tension on the natural frequency of microbeams. Applied Physics Letters, 90:231904, 2007. 